\title{
L'ingérence inattendue du Ministère de l'Enseignement Supérieur et de la Recherche Scientifique dans le recouvrement et la gestion des ressources propres des Universités publiques de Côte d'Ivoire
}

\author{
Dr KOFFI Fêtê Ernest \\ Sociologue, Enseignant-Chercheur
}

\begin{abstract}
RESUME
Les universités publiques ivoiriennes sont classées dans la catégorie des Etablissements Publics Nationaux (EPN). A ce titre, elles doivent générer des ressources propres et chercher des ressources additionnelles pour compléter les subventions que l'Etat leur octroie. Ces dernières années, l'on constate, non seulement la confiscation par l'Etat des $70 \%$ de frais de scolarité (engagement non respecté), mais l'ingérence inattendue de leur tutelle technique dans le recouvrement et la gestion de leurs ressources propres. Cette situation renvoie à l'épineuse et complexe question de l'autonomie des universités publiques ivoiriennes en général et particulièrement à leur gestion financière. Il s'avère nécessaire dans le contexte actuel, d'en débattre entre l'Etat et le monde universitaire pour tenir compte des préoccupations de chaque partie prenante, dans l'intérêt de l'enseignement supérieur ivoirien.
\end{abstract}

Mots clés : Etablissements Publics Nationaux (EPN), ressources propres, adéquation de locaux, dimensionnement des surfaces, ingérence et gestion financière

\begin{abstract}
Ivorian public universities are classified in the category of National Public Establishments (EPN). As such, they must generate their own resources and seek additional resources to supplement the subsidies that the State grants them. In recent years, it has been observed not only the confiscation by the State of $70 \%$ of tuition fees (commitment not respected), but the unexpected interference of their technical supervision in the recovery and management of their own resources. This situation refers to the thorny and complex issue of the autonomy of Ivorian public universities in general and particularly their financial management. In the current context, it is necessary to discuss it between the State and the academic world to take into account the concerns of each stakeholder, in the interest of Ivorian higher education
\end{abstract}

Keywords: National Public Establishments (EPN), own resources, suitability of premises, sizing of surfaces, interference and financial management

\section{Introduction}

L'école partout dans le monde est le milieu qui s'est toujours plaint de manque de moyens. Elle revendique sans cesse davantage d'infrastructures en quantité et en qualité et de ressources financières importantes.

Les Universités publiques Ivoiriennes s'inscrivent dans ces revendications. Elles ont chaque fois que l'occasion s'est présentée, dénoncé l'insuffisance des infrastructures, l'obsolescence des équipements et du manque de moyens financiers pour mener à bien leurs missions, à savoir l'enseignement, la recherche et la prestation de services.

Aujourd'hui, leurs besoins en infrastructures et en ressources financières se posent avec acuité. En effet lors de la crise postélectorale de 2010, leurs infrastructures ont été détruites et leurs matériels pillés 
systématiquement. Leur réhabilitation annoncée n'a mis en état que les infrastructures existantes dont les équipements et les laboratoires n'existent que de nom.

Il se pose à ces universités publiques les questions d'adéquation de locaux, du dimensionnement des surfaces, des équipements pédagogiques et numériques. C'est dans ce contexte que, face à la mondialisation et à la reconnaissance transfrontalière des diplômes, elles se sont engagées à adopter le système LicenceMaster-Doctorat (LMD).

Cette nouvelle architecture de l'enseignement supérieur, vise à assurer la qualité de la formation universitaire. Toutefois, son application et sa gestion nécessitent d'importants moyens matériels et financiers.

Dès lors, ces universités ne peuvent pas se contenter des budgets que l'Etat met à leur disposition chaque année, mais elles doivent recourir et renforcer la recherche des ressources propres que leur statut d'Etablissement Public National (EPN) leur impose de générer.

Mais, l'on constate aujourd'hui que le recouvrement de leurs ressources propres existantes ne s'opère pas aisément comme elles le voulaient. En effet, depuis une dizaine d'années, la tutelle (le ministère) les a dessaisies de cette prérogative et il lui revient désormais le rôle d'encaisser ces ressources avant de les leur verser.

Cette ingérence du ministère, selon nos enquêtés, met à mal la gestion financière de ces universités publiques qui doivent faire face à des grèves à répétition dues au non-paiement dans les délais, des indemnités allouées aux acteurs internes.

Dans ces conditions, il importe de fustiger cette immixtion de la tutelle dans la gestion des universités publiques, de nature à entraver le fonctionnement quotidien de ces établissements d'enseignement supérieurs qui renvoie à l'épineuse et délicate question de l'autonomie des universités de Côte d'Ivoire.

Trois parties constituent l'ossature de notre travail. La première est le cadre méthodologique de l'étude. La seconde porte sur la restitution des résultats et la troisième, la discussion.

\section{I-Methodologie}

\section{I-1 Type d'étude}

Nous inscrivons notre travail dans le cadre d'une étude qualitative. Nous avons eu recours à l'observation participante comme outils de collecte des données, comme un instrument de l'exploration théorique. Elle comprend deux types d'observations : l'observation qualitative et l'observation quantitative.

Ces deux types d'observations ne s'excluent pas mais elles se complètent, même si aujourd'hui, l'on note l'emprise des nombres dans les sciences sociales.

Nous avons eu aussi recours à la documentation et nous avons assisté à des conférences prononcées par des anciennes personnalités du monde universitaire

Nous avons agi non pas comme un enquêteur extérieur aux universités, mais comme un observateurparticipant. Pour obtenir les informations requises, nous avons vécu, vu, écouté, senti et touché dans l'exécution de nos taches et au cours des rencontres informelles.

Les informations retenues ici, ne résultent donc pas d'une enquête formelle, menée dans les règles de l'art. Mais, elles ont été recueillies à partir des visites de terrains.

\section{I-2 Site de l'étude}

Nos visites ont eu lieu dans les Universités Félix Houphouët Boigny (UFHB), Nangui Abrogoua (UNA), Alassane Ouattara (UAO).

Centres universitaires à l'origine suite à l'éclatement de l'Université Nationale de Côte d'Ivoire (1993), elles ont été érigées en universités à part entière en 1996. 


\section{I-3 Population cible}

Notre population visée a concerné principalement les agents des Directions des Scolarités Centrales (DSC) et ceux des services financiers à savoir la Direction des Affaires Financières et des Moyens Généraux (DAFMG), le Contrôle Budgétaire (CB) et l'Agence Comptable (AC).

Ces agents assermentés interviennent directement d'une manière ou d'une autre, dans le recouvrement des ressources propres des universités et dans la gestion des subventions de l'Etat.

$\mathrm{Si}$ au cours de nos visites, certains agents n’ont pas voulu nous révéler clairement les désagréments que cause l'immixtion du ministère dans le recouvrement et la gestion des ressources propres des universités publiques, beaucoup d'autres ont libéré les informations et mis à notre disposition des données qui nous ont permis d'obtenir les résultats que nous présentons ci-après.

\section{II-Resultats}

\section{II-1 Origine des ressources propres des universités publiques.}

Les universités publiques ivoiriennes sont classées dans la catégorie des Etablissements Publics Nationaux(EPN). Selon la loi ${ }^{\circ} 98-338$ du 02/07 /1998, un EPN est une personne morale de droit public créé par l'Etat, disposant de l'autonomie financière dont l'objet exclusif et spécialisé, est de remplir une mission de service public, en suivant des règles adaptées à la mission et comptant des contraintes et de prérogatives de droit public.

Avant tout, il convient d'indiquer que le budget des universités publiques est constitué essentiellement de subventions de 1'Etat qui représentent en moyenne 93\% contre seulement 7\% pour les ressources propres.

Ces universités ont bénéficié d'environ 86695033706 de francs CFA pour la couverture de leurs budgets en 2020 et de 88953090972 de francs CFA.

Initialement ces budgets étaient constitués de deux types de ressources : La dotation ou la subvention de l'Etat constitue leurs budgets principaux qui comprennent une partie (Titre I) destinée au fonctionnement et l'autre (Titre II) prévue pour l'investissement.

Cette dernière partie a disparu après leur réhabilitation à l'identique (pas de nouvelles constructions) suite aux dégâts qu'elles ont subis lors de la crise postélectorale de 2010. Les budgets énoncés ci-dessus comprennent donc le titre I (budget de fonctionnement) et les ressources propres générées par les universités elles-mêmes qui tournent autour de 6.043.139.520 FCFA en 2020 et de 6.182. 830.000 de FCFA en 2021

\section{BUDGETS DES UNIVERSTES PUBLIQUES DE COTE D'IVOIRE 2020 ET 2021}

\begin{tabular}{|c|c|c|c|c|c|}
\hline \multirow{2}{*}{ UNIVERSITES } & \multicolumn{3}{|l|}{ BUDJET 2020} & \multicolumn{2}{|l|}{ BUDJET 2021} \\
\hline & SE & $\mathbf{R P}$ & TOTAL & SE & $\mathbf{R P}$ \\
\hline UFHB & 44078931067 & 3219400000 & 47298331067 & 42834634899 & 3350000000 \\
\hline UNA & 7815946765 & 751909520 & 8567856285 & 8510974905 & 450000000 \\
\hline UAO & 13726958996 & 1050000000 & 14776958996 & 14192227776 & 1250000000 \\
\hline UJLOG & 5298790849 & 370830000 & 5669620849 & 5738800938 & 370830000 \\
\hline UPGC & 6749891633 & 351000000 & 7100891633 & 7722889168 & 342000000 \\
\hline UM & 1973374876 & 50000000 & 2023374876 & 2239294302 & 70000000 \\
\hline UVCI & 1008000000 & 250000000 & 1258000000 & 1531438984 & 350000000 \\
\hline TOTAL & 80651894186 & 6043139520 & 86695033706 & 82770260972 & 6182830000 \\
\hline
\end{tabular}

SE : Subventions de l'Etat

RP : Ressources propres

Les ressources propres des universités publiques ivoiriennes comprennent les taxes universitaires acquittées par les étudiants. Il s'agit ici, essentiellement des frais des préinscriptions qui s'adressent aux bacheliers de l'année en cours, d'inscriptions qui concernent tous ceux qui s'inscrivent pour la première fois dans l'une des universités du pays et de réinscriptions qui prennent en compte les anciens étudiants ayant actualisé leur situation suivant leurs résultats de fin d'année (admis ou redoublants). 
Les fonds tiers qui sont alimentés par des mandats de recherche privés et publics, des donations et des legs affectés à des buts précis et qui devraient augmenter les ressources, sont pratiquement inexistants dans les universités publiques. Il est à indiquer de 1975 à 2010 les taxes étaient fixées comme suit : premier cycle $6000 \mathrm{~F}$, second cycle $8000 \mathrm{~F}$ et le troisième cycle $10.000 \mathrm{~F}$.

Avec l'entrée en vigueur de la réforme L.M.D et face aux exigences matérielles et financières de sa mise en œuvre, la conférence des présidents des universités et grandes écoles publiques a demandé et obtenu la hausse des frais de scolarité dans les universités publiques.

C'est ainsi qu'avec l'aval du gouvernement les frais de scolarité sont passés de $6000 \mathrm{f}$ à $100.000 \mathrm{f}$ pour le premier cycle (Licence) de $8000 \mathrm{f}$ à $200.000 \mathrm{f}$ pour le second cycle (Master) et de $10.000 \mathrm{f}$ à $300.000 \mathrm{f}$ pour le troisième cycle (Doctorat).

Les ressources d'un EPN peuvent être subsidiairement constituées par des dons et legs acceptés conformément aux dispositions en vigueur, des dotations et subventions de l'Etat et des subventions des organismes nationaux et internationaux.

Dans ces universités publiques ivoiriennes, l'on note que les mandats de recherches privées ou publiques, des donations et des legs n'existent pratiquement pas. Aussi les collectivités locales interviennent-elles rarement dans le financement des prestations de base nécessaires à l'enseignement supérieur et à la recherche.

\section{II-2 Immixtion de la Tutelle dans le recouvrement des ressources propres des universités publiques}

Comme indiqué plus haut, les frais de scolarité ont connu une augmentation. Mais cette hausse de taxes universitaires intervient au moment où le pays sortait de la grave crise post-électorale de 2010, aux conséquences durement ressenties par les parents d'élèves.

Ceux-ci ont exprimé au gouvernement leurs difficultés financières du moment qui a accepté de prendre en charge pendant trois ans soixante-dix pour cent (70\%) des différents nouveaux frais fixés. Durant cette période de trois années les étudiants de Licence payent $30.000 \mathrm{f}$, ceux du Master $60.000 \mathrm{f}$ et les Doctorants $90.000 \mathrm{~F}$.

Il y a lieu de relever que pour des raisons non encore révélées le gouvernement n'a jamais respecté son engagement, celui de verser aux universités les $70 \%$ des frais de scolarité, les plongeant de la sorte dans une situation financière sombre.

Aussi, le délai des trois ans est-il passé et ni le gouvernement ni la conférence des présidents des universités et grandes écoles publiques n'osent revenir sur les frais initialement fixés à savoir les $100.000 \mathrm{f}$, les 200.000 $\mathrm{f}$ et les $300.000 \mathrm{f}$.

Le comble est que la gestion de ces frais qui constituent les ressources propres des universités échappe à ces dernières. Les préinscriptions sont une opération au cours de laquelle les nouveaux bacheliers expriment leur désir de poursuivre leurs études dans l'enseignement supérieur.

Cette opération était menée par les universités sous la supervision de la DESUP. Depuis l'année 2013-2014, cette opération est directement pilotée par le MESRS. Les universités n'y sont même pas associées et leurs services financiers ne savent pas où vont les frais générés par ladite opération.

Par ailleurs lorsque les bacheliers sont retenus dans les universités chacun s'inscrit en ligne en payant les frais de scolarité (30.000 FCFA) via les téléphonies mobiles qui les versent dans un compte ouvert à l'insu des universités et logé au MESRS pour être, dit-on, reversés par la suite aux universités. Si par chance une université perçoit un reversement, son montant n'est pas accompagné d'un état pour permettre de savoir à quel effectif d'étudiants correspond-il.

Au cours d'une conférence de presse, l'ancien ministre de l'enseignement supérieur et certains enseignants dans un journal de la place ont révélé certaines choses. Monsieur Gnamien Konan ex-DG de la douane est 
un sachant. Il a été successivement Ministre de la fonction publique et de la réforme administrative, Ministre de l'enseignement Supérieur et de la Recherche Scientifique et Ministre de l'habitat et du logement social.

Il déclare sans ambages face à la nation ivoirienne que « des Ministres utilisent l'argent d'inscriptions et de concours pour financer leur parti politique. Que ce soit les frais de concours, les frais d'orientation, les frais d'inscription dans les universités, c'est une vaste escroquerie organisée par les Ministres pour financer leurs propres activités ou pour financer leurs partis politiques.

Vous verrez que ceux qui font cela sont de grands dirigeants et qui ont de hautes responsabilités. L'argent ne va pas au budget. Moi, je n'ai pas vu de traces au budget. Je suis passé à la fonction publique, avant moi, c'était le cas. Après moi c'est le cas. A l'Enseignement Supérieur c'est la même chose », il a conclu en affirmant que «les frais annexes et de concours sont une arnaque ».

Suite à cette intervention de l'ex-Ministre de l'ESRS, un groupe d'enseignants du supérieur a démontré avec un tableau à l'appui que les ressources propres des Universités sont détournées et a dénoncé le MESRS d'être le « fossoyeur » des Universités.

Selon ces enseignants «tout bachelier débourse $10.000 \mathrm{f}$ pour se préinscrire. A la rentrée scolaire 20162017, 60.000 bacheliers se sont préinscrits en raison de $10.000 \mathrm{f}$, ce qui a permis au MESRS d'empocher 600.000 000FCFA. Cette somme revenait de droit aux universités, parce que constituant une partie de leurs ressources propres.

Les mêmes enseignants dans un tableau reproduit ci-dessous, ont relevé que l'argent que les Universités devaient percevoir à travers les inscriptions mais qui est géré par le MESRS équivaut à plus ou moins trois milliards au titre de l'année universitaire ci-dessus indiquée.

\begin{tabular}{|l|l|lc|l|}
\hline Niveaux d'études & EFFECTIFS ETUDIANTS & $\begin{array}{l}\text { FRAIS } \\
\text { SCOLARITE }\end{array}$ & $\begin{array}{l}\text { RESSOURCES } \\
\text { ATTENDUES }\end{array}$ \\
\hline LICENCE & 45000 & 30.000 & 1.350 .000 .000 \\
\hline MASTER & 20000 & 60.000 & 1.200 .000 .000 \\
\hline DOCTORAT & 5000 & 90.000 & 450.000 .000 \\
\hline TOTAL & 70.000 & --- & 3.000 .000 .000 \\
\hline
\end{tabular}

Les sources de ces données n'ont pas été révélées. Même s'il faut les prendre avec circonspection, l'on constate que les Universités publiques ne maîtrisent plus leurs ressources propres. Les services de scolarité éprouvent des difficultés à communiquer des statistiques exactes du fait que les inscriptions s'étendent sur la toute la longueur de l'année.

Les agents comptables, les contrôleurs budgétaires et les DAFMG ne parviennent pas à maitriser les effectifs des étudiants ayant effectué le paiement des frais de scolarité. Ils informent, disent-ils, leurs hiérarchies de cette gestion de fait, mais celles-ci ne réagissent pas comme si elles approuvent ce détournement déguisé.

Curieusement, face à ces révélations, ni le MESRS, ni le Ministère de l'Economie et des Finances, ni le Ministère du Budget, en somme le Gouvernement ne réagit pas pour éclairer l'opinion nationa

\section{Discussion}

Les universités publiques ivoiriennes, inscrites dans cette catégorie des Etablissements Publics Nationaux (EPN) sont en droit de générer des ressources propres et chercher des ressources additionnelles dans l'accomplissement des leurs missions.

En tant qu'EPN, elles sont placées sous la tutelle économique et financière du ministre chargé de l'Economie et des Finances et sous la tutelle technique du ministre de l'enseignement supérieur et de la recherche scientifique. 
Chaque université est placée sous le contrôle et l'autorité d'un Conseil de gestion, qui suit, de façon permanente, la bonne exécution de ses missions et contrôle la préparation et l'exécution du budget et examine le compte financier produit par l'agent comptable en fin d'exercice. Elle a en son sein, un Agent Comptable (AC), un Contrôleur Budgétaire (CB) et un Directeur des Affaires Financières et des Moyens Généraux (DAFMG). Le Président est l'ordonnateur principal et le Secrétaire Général est l'ordonnateur en second.

Alors, avec de telles dispositions, comment comprendre cette ingérence inattendue du ministère technique dans le recouvrement et la gestion des ressources propres qu'elle est habilitée à générer ?

Au ministère, il nous est relevé qu'il y a de bonnes raisons que l'Etat intervienne dans ces universités : en tant que financeur, il se réserve le droit de contrôler l'utilisation des fonds publics et de corriger le comportement des universitaires. En plus, l'Etat est le responsable de la protection du public, à ce titre, il doit garantir des diplômes et s'assurer de la qualité des prestations universitaires.

Aussi, l'Etat ivoirien subventionne-t-il à plus de $98 \%$ les universités, par conséquent, il a un droit de regard dans leur gestion. Elles restent assujetties aux lois et règlements fixés par celui-ci.

Les pouvoirs insistent sur le fait que les universités publiques sont des institutions dans lesquelles l'Etat « engloutit » beaucoup de ressources matérielles et financières, eu égard aux missions à elles assignées pour contribuer au processus de développement national. Par conséquent, il est hors de question que ces dernières fonctionnent en vase clos. Ainsi, comme il a été relevé précédemment, le titre II de leurs budgets relatifs à l'investissement est géré par une structure externe qui a exécuté la réhabilitation des universités après la crise postélectorale de 2010 d'une part et les ressources propres que ces universités doivent générer leur échappent d'autre part.

Quant à la gestion du titre I affecté à leur fonctionnement, elle trouble le sommeil des ordonnateurs à la proche des fins de mois ou des fins de trimestres, pendant les périodes de paiements des salaires, des primes de recherche, des heures supplémentaires, des heures de vacations et des fournisseurs.

Etant donné que les ressources propres de leurs institutions n'existent pratiquement pas, ils sont toujours exposés à la fronde des travailleurs, en ce sens que tant que la tutelle économique, à savoir le Ministre chargé du budget en liaison avec le Ministre chargé de l'économie et des Finances n'approvisionne pas les comptes des universités, ils ne peuvent pas payer ce qui revient de droit à ces travailleurs.

Un autre mode de gestion financière est instauré dans les procédures de passation des marchés : «l'appel d'offre ». Cette procédure, qui consiste à centraliser tous les besoins aux Directions des Affaires Financières et des Moyens Généraux (DAFMG) chargées de trouver les «moins disant », perturbe le fonctionnement des universités.

En effet, avec ce mode de gestion centralisé, il n'est pas certain que les matières d'œuvre, à savoir les produits chimiques et les fournitures diverses s'obtiennent pendant l'année au cours de laquelle l'appel d'offre est lancé.

Ainsi, les Présidents de nos universités sont confrontés à un ensemble de contraintes qui limitent leurs capacités d'agir

Devant une telle situation, comment les universités publiques ivoiriennes, ne maîtrisant pas leurs ressources additionnelles et qui ne se contentent que de la subvention de l'Etat, peuvent-elles affronter les exigences de la reforme LMD, surtout en matière d'infrastructures appropriées et de ressources financières conséquentes ?

C'est ici que surgit l'épineuse question de l'autonomie des universités publiques. Le débat sur l'autonomie des universités publiques n'est pas nouveau dans les milieux Universitaires du monde. C'est une question assez complexe qui part de l'histoire des rapports tumultueux et complexes entre la Science, la Politique et l'Eglise, l'idée étant de mettre l'université à l'abri de certains “confinements' religieux ou politiques. 
Dans les pays de longues traditions universitaires, une université autonome, dispose aujourd'hui, de tous les leviers pour organiser ses formations en fonction des besoins de ses étudiants et de la situation de l'emploi, recruter des chercheurs de haut niveau, valoriser l'engagement des personnels, créer des fondations, développer les coopérations avec les entreprises.

En somme l'autonomie de l'université libère les énergies, renforce sa réactivité et lui donne plus de légitimité face à leurs partenaires : une université peut bâtir un projet d'établissement, faire des choix stratégiques, mener une véritable politique de recrutement, gérer un budget global, assurer une nouvelle mission d'insertion professionnelle des étudiants. Il s'agit d'améliorer les conditions de vie et de travail de l'ensemble de la communauté universitaire, de renforcer les formations pour une meilleure réussite des étudiants et de développer l'attractivité des métiers de l'enseignement supérieur et de la recherche.

En Côte d'Ivoire, ces dispositions semblent se présenter à nos universités comme une utopie. Ces dernières années, il est donné de constater que les ordonnateurs de ces universités rencontrent de réelles difficultés à gérer les budgets qui leur sont alloués. Selon ceux-ci et les responsables des services financiers, ils ne maîtrisent plus la gestion de ces budgets.

Les acteurs universitaires internes n'admettent pas que l'Etat conserve le monopole de la définition des principes généraux dans le domaine de la formation et particulièrement dans la gestion des ressources financières. Cette immixtion de l'Etat dans les universités publiques, ne doit pas être trop contradictoire avec les traits professionnels de l'organisation universitaire.

Dans le contexte actuel, marqué par la limitation des ressources étatiques, l'explosion des effectifs des étudiants et l'instauration du LMD dans l'enseignement supérieur, les universités publiques doivent cesser d'être totalement tributaires des décisions publiques. Il faut leur donner des possibilités de définir des stratégies et conduire des politiques de gestion de leurs ressources financières en recherchant elles-mêmes des ressources matérielles et financières pour affronter les exigences de l'application de la réforme LMD en leur sein.

Par conséquent, il est plus qu'une nécessité que ces universités obtiennent de l'Etat une autonomie de gestion financière. Les Ministères du Budget et de l'Economie et des Finances, qui assurent leur tutelle économique doivent leur déléguer des prérogatives et des responsabilités en matière de dépenses pour leur permettre d'instaurer de nouvelles procédures de gestion de leurs ressources.

Il importe que les autorités académiques, le Ministre de l'enseignement Supérieur et le Ministre chargé du budget en liaison avec le Ministre chargé de l'économie et des Finances engagent un débat franc pour donner un contenu à l'autonomie, devant prendre en compte les préoccupations de chaque partie prenante en insistant sur l'autonomie de gestion financière qui renvoie au financement des universités publiques.

\section{Conclusion}

Les universités publiques ivoiriennes qui, face à la mondialisation et à la reconnaissance transfrontalière des diplômes, se sont engagées à adopter le système Licence-Master-Doctorat (LMD) sont à la recherche de ressources matérielles et financières pour l'adoption réussie de cette nouvelle architecture de l'enseignant supérieur.

C'est en ce moment-là que la tutelle technique (MESRS) signe, de façon inattendue, son ingérence dans le recouvrement et la gestion des ressources qu'elles sont censées générer pour compléter les subventions qu'elles perçoivent de l'Etat.

En effet, depuis 2012, le gouvernement s'étant engagé de payer aux universités les $70 \%$ des nouveaux frais de scolarité, obtenus difficilement par la conférence des Présidents des universités et grandes publiques de Côte d'Ivoire (100.000, 200.000 et 300.000 FCFA), n'a pas encore honoré son engagement.

Par ailleurs, le Ministère confisque les $30 \%$ de ces frais que les parents d'étudiants paient qui leur sont destinés. IL recouvre les frais de scolarité (30.000 FCF, 60.000 FCFA et 90.000 FCFA) via les téléphonies 
mobiles qui les versent dans un compte ouvert à l'insu des universités et logé au MESRS pour être, dit-on, reversés par la suite aux universités. Si une université perçoit un reversement, son montant n'est pas accompagné d'un état pour permettre de savoir à quel effectif d'étudiants il correspond.

Le constat est que les universités publiques de côte d'ivoire sont «spoliées » de leurs ressources propres, constituant leurs appuis budgétaires, au vu et au su de tous les acteurs internes d'une part, et le gouvernement semble être complice de cette spoliation des institutions universitaires d'autre part. Devant une telle situation qui trouble le sommeil de leurs ordonnateurs à la veille du payement des différentes primes, comment ces universités publiques ivoiriennes, «dépouillées » de leurs ressources additionnelles et qui ne comptent que sur les subventions de l'Etat, peuvent-elles affronter les exigences de la reforme LMD, surtout en matière d'infrastructures appropriées et de ressources financières conséquentes ? Il apparait nécessaire que les Ministères du Budget et de l'Economie et des Finances, qui assurent leur tutelle économique leur délèguent des prérogatives et des responsabilités en matière de dépenses pour leur permettre d'instaurer de nouvelles procédures de gestion de leurs ressources.

\section{Bibliographie}

1. Assa Ayemu, Bakayoko-Ly Ramata, Kttié A.Louka (2003). Projets de recherche. De 1 conception u montage et au financement. Que faire faire ? Abidjan, Editions Universitaires de Côte d'Ivoire

2. Banque mondiale (1994). L'enseignement supérieur

3. Cazenave Ph. 1994, Table ronde «Politique de l'enseignement supérieur et crise du financement», Revue Politiques et management public

4. Ernest Fêtê KOFFI (2017). La GOUVERSNANCE DES UNIVERSITES PUBLIQUES DE COTE D'IVOIRE : Etude rétrospective (2000-2010) des perceptions des acteurs internes de l'Université d'Abobo-Adjamé . Collection : Essai EDUCI

5. Michaela Martin (2014). LA GOUVERNANCE DANS L'ENSEIGNEMENT SPUERIEUR : quelles politiques, avec quels défis ? IIPE UNESCO.

6. Loi $\mathrm{n}^{\circ}$ 98-388 du 02/07/1998 fixant les règles générales relatives aux Etablissements Publics Nationaux (EPN) et portant création de catégories d'établissements publics et abrogeant la loi $n^{\circ} 80$ $1070 \mathrm{du} 13 / 09 / 1980$

7. Ministère de l'Education Nationale (1991). Université 2000: quelle université pour demain ? PARIS ? La documentation

8. Ruben Alvarez et Claude Echevin (1999). Revue Politiques et management public

9. Rida Lamrani (2007). L'Université marocaine, autrement: a world class university : Essai, marsam.

10. Sawadogo. S. (1995). L'avenir des universités africaines: Mission et rôle. Assouciation des Universités Africaines 